\title{
Semi-lagrangian Semi-implicit Fully Splitted Hydrostatic Atmospheric Model
}

\author{
Andrei Bourchtein \\ Pelotas State University, Department of Mathematics \\ Rua Anchieta 4715 bloco K, ap.304, Pelotas 96020-250, Brazil \\ bursteinaterra.com.br
}

\begin{abstract}
Different splitting techniques are applied in the context of semiLagrangian semi-implicit approach to develop computationally efficient scheme. Vertical decoupling permits to apply simpler explicit calculations for slow vertical modes and transforms the $3 \mathrm{D}$ elliptic equation arising on each time step of the scheme to a set of $2 \mathrm{D}$ elliptic problems. By time splitting, the last are reduced to 1D problems, which admit an efficient direct solver. Finally, the space splitting diminishes the amount of operations due to approximation of the physically insignificant terms with lower order of accuracy. Application of these techniques results in an efficient scheme with time step chosen according to accuracy consideration and the amount of calculations proportional to the number of spatial grid points. Performed numerical experiments show good computational performance of the algorithm and accuracy of forecasting meteorological fields.
\end{abstract}

\section{Introduction}

In atmospheric simulations based on hydrostatic equations, the physically significant processes have frequently much lower frequency than the highest frequency motions admitted by primitive system, such as high-frequency gravity waves and strong local advective processes. The numerical schemes used to integrate the primitive equations are often time-step limited by Courant-Friedrichs-Lewy condition with respect to the fastest processes treated explicitly. Therefore explicit approximation of fast, physically irrelevant modes can imply excessive restriction on time step, such that time truncation errors are some orders smaller than that due to spatial discretization for the motions of meteorological interest. One of the most widespread techniques in atmospheric modeling, which permits to circumvent both gravity wave and advection restrictions on the time step, is semi-Lagrangian semi-implicit (SLSI) approach introduced by Robert [19]. In such schemes the time step is limited essentially by Pudykiewicz-Benoit-Staniforth condition, which allows to use the time steps up to 1 hour in the fine grid models $[17,22]$. This means that the time step can be primarily chosen on the basis of accuracy consideration. Besides, in some SLSI schemes, the number of operations required for each time step is directly proportional to the number of spatial grid points, that is, these SLSI schemes are economic ones. Different variants of SLSI schemes have been developed in context of mesoscale, 
regional and global models and have been successfully applied for numerical weather prediction and general atmospheric circulation [3, 11, 15, 18, 24].

In spite of many attractive properties of SLSI approach, there are still different problems to be solved, in the first place, to improve the treatment of the implicit part of the SLSI schemes. The purpose of this study is to develop computationally efficient and precise SLSI model of regional weather prediction. For this, we apply two additional splitting techniques to implicit part of the SLSI scheme. The first is vertical splitting by Burridge [6], which permits to decouple 3D problem in the set of the 2D ones. By this way, a 3D elliptic equation needed to be solved at each time step is replaced by a set of $2 \mathrm{D}$ Helmholtz equations. Moreover, this vertical decoupling give a possibility to treat implicitly only the fastest vertical modes and other ones can be approximated explicitly and, consequently, in a simpler manner.

The reduction of $3 \mathrm{D}$ elliptic problem to a set of $2 \mathrm{D}$ ones is a significant simplification, but the last problems are still costly to solve. To overcome this difficulty, we use the time splitting technique proposed by Tanguay and Robert [23] to factorize 2D Helmholtz equation in a set of $1 \mathrm{D}$ problems, which are solved very effectively by direct Gelfand-Thomas algorithm. Finally, to reduce the amount of calculations for explicit part of the SLSI scheme, we use the space splitting by Turkel and Zwas [25] to approximate the physically insignificant motions with a lower order of accuracy.

The paper is organized in the following way. In section 2 we present the primitive equations of the hydrostatic atmospheric model. The algorithm of the developed fully splitted SLSI scheme is described in section 3. The results of numerical experiments and discussion of the scheme performance are presented in section 4 , following by concluding remarks in section 5 .

\section{Continuous Equations}

The prognostic equations of the hydrostatic atmospheric model consist of horizontal momentum equations, continuity equation and thermodynamic equation. Choosing Cartesian coordinates $x, y$ of a conformal mapping projection and vertical coordinate $\sigma=p / p_{s}$, these equations can be written as follows:

$$
\frac{d u}{d t}=N_{u}-G_{x}, \frac{d v}{d t}=N_{v}-G_{y}, \frac{d P}{d t}=-D-\dot{\sigma}_{\sigma}, \frac{d T}{d t}=N_{T}+\frac{R T_{0}}{c_{p}}\left(\frac{d P}{d t}+\frac{\dot{\sigma}}{\sigma}\right),
$$

where $N_{u}, N_{v}, N_{T}$ are the nonlinear and variable coefficient terms:

$$
\begin{gathered}
N_{u}=-\frac{u^{2}+v^{2}}{2} m_{x}^{2}+f v-R\left(T-T_{0}\right) P_{x}, \\
N_{v}=-\frac{u^{2}+v^{2}}{2} m_{y}^{2}-f u-R\left(T-T_{0}\right) P_{y}, N_{T}=-\frac{R\left(T-T_{0}\right)}{c_{p}}\left(\frac{\dot{\sigma}}{\sigma}-D-\dot{\sigma}_{\sigma}\right) .
\end{gathered}
$$

The system is closed by adding two diagnostic equations: hydrostatic and vertical velocity ones 


$$
G_{\ln \sigma}=-R T, \dot{\sigma}=\sigma \int_{0}^{1} D+m^{2}\left(u P_{x}+v P_{y}\right) d \sigma-\int_{0}^{\sigma} D+m^{2}\left(u P_{x}+v P_{y}\right) d \sigma
$$

and by imposing the upper and lower boundary conditions: $\dot{\sigma}=0$ at $\sigma=0$ and 1 . (The vertical velocity equation can be derived from continuity equation and boundary conditions). Here,

$$
\frac{d \varphi}{d t}=\varphi_{t}+m^{2}\left(u \varphi_{x}+v \varphi_{y}\right)+\dot{\sigma} \varphi_{\sigma}
$$

is the 3D individual derivative, $u$ and $v$ are the horizontal velocity components and the following common denotations are used: $p$ and $p_{s}$ are the pressure and surface pressure respectively, $P=\ln p_{s}, t$ is the time, $m$ is the mapping factor, $f$ is the Coriolis parameter, $D=m^{2}\left(u_{x}+v_{y}\right)$ is the horizontal divergence, $T$ is the temperature, $T_{0}=$ const is the reference temperature profile, $G=\Phi+R T_{0} P$, $\Phi=g z$ is the geopotential, $z$ is the height, $g$ is the gravitational acceleration, $R$ is the gas constant, $c_{p}$ is the specific heat at constant pressure. The subscripts $t, x, y, \sigma$ denote the partial derivatives with respect to indicated variable.

\section{Design of Semi-lagrangian Semi-implicit Scheme}

In this section we describe economic algorithm for solution of the above equations. The spatial grid is composed of staggered horizontal $C$ grid with meshsize $h=75 \mathrm{~km}$ and staggered vertical Lorenz grid with $K=15$ levels [1, 4, 14]. In subsequent description we will use the model vertical structure matrix $\mathbf{A}$, which is uniquely determined by the choice of vertical levels, reference temperature profile and difference approximation of vertical derivatives in hydrostatic, continuity and thermodynamic equations. Therefore this matrix does not depend on prognostic values and can be calculated only once before forecasting. In [5] it was shown that this matrix has distinct positive eigenvalues and, therefore, it has real spectral decomposition $\mathbf{A}=\mathbf{S} \Lambda \mathbf{S}^{-1}$, where $\Lambda=\operatorname{diag}\left[\lambda_{1}, \ldots, \lambda_{K}\right]$ is the diagonal eigenvalue matrix and $\mathbf{S}$ is the matrix of eigenvectors (i.e., vertical normal modes). The details of the used horizontal and vertical grids can be found in $[4,5]$.

Each time step of the developed SLSI splitted scheme consists of the successive segments of computation, which we describe in the following subsections.

\subsection{Semi-lagrangian Explicit Scheme}

First, we evaluate the preliminary prognostic values by semi-Lagrangian explicit (SLEX) scheme (only prognostic equations are presented): 


$$
\begin{gathered}
\frac{\hat{u}^{n+1, a}-u^{n-1, d}}{2 \tau}=\frac{N_{u}^{n, a}+N_{u}^{n, d}}{2}-\frac{G_{x}^{n, a}+G_{x}^{n, d}}{2}, \\
\frac{\hat{v}^{n+1, a}-v^{n-1, d}}{2 \tau}=\frac{N_{v}^{n, a}+N_{v}^{n, d}}{2}-\frac{G_{y}^{n, a}+G_{y}^{n, d}}{2}, \\
\frac{\hat{P}^{n+1, a}-P^{n-1, d}}{2 \tau}=-\frac{D^{n, a}+D^{n, d}}{2}-\frac{\dot{\sigma}_{\sigma}^{n, a}+\dot{\sigma}_{\sigma}^{n, d}}{2}, \\
\frac{\hat{T}^{n+1, a}-T^{n-1, d}}{2 \tau}=\frac{N_{T}^{n, a}+N_{T}^{n, d}}{2}+\frac{R T_{0}}{c_{p}}\left(\frac{\hat{P}^{n+1, a}-P^{n-1, d}}{2 \tau}+\frac{\dot{\sigma}^{n, a}+\dot{\sigma}^{n, d}}{2 \sigma}\right) .
\end{gathered}
$$

Hereinafter, $\tau$ is the time step, superscripts " $n+1, a$ " denote a value at the arrival point of the $3 \mathrm{D}$ trajectory at the new time level $(n+1) \tau$, superscripts " $n-1, d$ " denote a value at the departure point of the trajectory at old time level $(n-1) \tau$ and superscripts " $n, a$ " and " $n, d$ " denote the values at the arrival and departure points at current time level $n \tau$. Arrival points are the given points of the regular spatial grid and the departure points are calculated by usual iterative procedure with second order of accuracy $[13,20]$. The sufficient convergence condition for these iterations is the inequality

$$
\tau \leq 1 / 3 V_{d}, \quad V_{d}=m^{2} \max \left(\left|u_{x}\right|,\left|u_{y}\right|,\left|u_{\sigma}\right|,\left|v_{x}\right|,\left|v_{y}\right|,\left|v_{\sigma}\right|,\left|\dot{\sigma}_{x}\right|,\left|\dot{\sigma}_{y}\right|,\left|\dot{\sigma}_{\sigma}\right|\right),
$$

which represents the generalization of the condition by Pudykiewicz et al. to the case of 3D trajectories [4]. For our spatial grid $V_{d} \approx 1.5 \cdot 10^{-4} \mathrm{~s}^{-1}$ and maximum allowable time step is $\tau \approx 40 \mathrm{~min}$.

The modified tricubic interpolation is used to calculate the values $u^{n-1, d}$ and $N_{u}^{n, d}$ at the departure points $u$, the values $v^{n-1, d}$ and $N_{v}^{n, d}$ at the departure points $v$ and the terms $P^{n-1, d}, T^{n-1, d}$ and $N_{T}^{n, d}$ at the departure points $T$ (the details of the modified or quasi-tricubic interpolation can be found in [21]). Finally, the values of $G_{x}{ }^{n, d}, G_{y}{ }^{n, d}$ and $D^{n, d}, \dot{\sigma}^{n, d}$ are obtained by trilinear interpolation at the departure points $u, v$ and $T$ respectively. The higher order interpolation is not applied to the latter terms because their principal vertical components will be corrected on the next segment of computations. Moreover, the central differences with triple space step $h_{g}$ on grid $C$ are used to approximate the spatial derivatives of the gravity wave terms $G_{x}, G_{y}, D$. Since meshsize $h / 2=37.5 \mathrm{~km}$ is used for the best approximation of gravity terms on grid $C$, the triple meshsize $h_{g}$ is equal to $112.5 \mathrm{~km}$. Having calculated all the terms at time levels $n-1$ and $n$, we can find the unknowns $\hat{u}^{n+1, a}, \hat{v}^{n+1, a}, \hat{P}^{n+1, a}, \hat{T}^{n+1, a}$ by simple explicit formulas (5).

Hydrostatic and vertical velocity diagnostic equations are approximated with second order accuracy at each time level. 


\subsection{Vertical Decoupling and Correction Equations}

The second segment is preparation for computation of corrections to preliminary explicit prognostic values. First, we calculate right-hand sides for correction equations by formulas

$$
\widetilde{\varphi}=\left(\hat{\varphi}^{n+1, a}-\varphi^{n, a}\right)-\left(\varphi^{n, d}-\varphi^{n-1, d}\right), \varphi=u, v, D, G .
$$

Vertically dicretized physical functions (7) can be transformed to its vertical mode coefficients by formulas

$$
\varphi^{*}=\mathbf{S}^{-1} \tilde{\varphi}, \varphi=\mathbf{u}, \mathbf{v}, \mathbf{D}, \mathbf{G} .
$$

Then we find an approximate solution of the following 2D systems for corrections

$$
u_{k}+\tau G_{k_{x}}=-\tau G_{k_{x}}^{*}, v_{k}+\tau G_{k y}=-\tau G_{k y}^{*}, G_{k}+\tau c_{k}^{2} D_{k}=-\tau c_{k}^{2} \cdot D_{k}{ }^{*},
$$

where $k=1, \ldots, I$ is the number of corrected vertical modes, $c_{k}^{2}=R T_{0} \lambda_{k}$ is the square of the gravity-wave phase speed of the $k$-th vertical mode and $u_{k}, v_{k}, D_{k}, G_{k}$ are the vertical mode corrections. Hereinafter we suppose that eigenvalues $\lambda_{k}$ are numbered in decreasing order.

One can show that if $I=K$, then the solution of (9) is equivalent to the solution of the traditional three-time-level SLSI scheme [20, 22]

$$
\begin{gathered}
\frac{u^{n+1, a}-u^{n-1, d}}{2 \tau}=\frac{N_{u}^{n, a}+N_{u}^{n, d}}{2}-\frac{G_{x}^{n+1, a}+G_{x}^{n-1, d}}{2}, \\
\frac{v^{n+1, a}-v^{n-1, d}}{2 \tau}=\frac{N_{v}^{n, a}+N_{v}^{n, d}}{2}-\frac{G_{y}^{n+1, a}+G_{y}^{n-1, d}}{2}, \\
\frac{P^{n+1, a}-P^{n-1, d}}{2 \tau}=-\frac{D^{n+1, a}+D^{n-1, d}}{2}-\frac{\dot{\sigma}_{\sigma}^{n+1, a}+\dot{\sigma}_{\sigma}^{n-1, d}}{2}, \\
\frac{T^{n+1, a}-T^{n-1, d}}{2 \tau}=\frac{N_{T}^{n, a}+N_{T}^{n, d}}{2}+\frac{R T_{0}}{c_{p}}\left(\frac{P^{n+1, a}-P^{n-1, d}}{2 \tau}+\frac{\dot{\sigma}^{n+1, a}+\dot{\sigma}^{n-1, d}}{2 \sigma}\right),
\end{gathered}
$$

where the modified tricubic interpolation has been used for all terms at the departure points.

To preserve the accuracy and stability of the scheme it is sufficiently to solve only some first systems (9) with highest gravity-wave phase speeds. Really, the analysis of linear stability showed that using coarser meshsize $h_{g}=112.5 \mathrm{~km}$ for approximation of pressure gradient and divergence terms we will reach the same time step as in SLSI scheme if the systems with $c_{k}>33 \mathrm{~m} / \mathrm{s}$ are solved. For used model specifications, the gravity-wave phase speeds are $\left\{c_{k}\right\}_{1}^{15}=\{342,188,101,61.8,41.6,29.8,22.1,16.7,12.7,9.6,7.2,5.2,3.6,2.3,1.1\}$, which implies that only corrections for the first five modes have to be found by solving (9) and other vertical modes can be treated explicitly. We will refer to this 
scheme as SLSI-V (semi-Lagrangian semi-implicit vertically splitted) scheme. Theoretically this scheme has the first order accuracy because of linear interpolation of the gravity wave terms $G_{x}{ }^{n, d}, G_{y}{ }^{n, d}, D^{n, d}, \dot{\sigma}^{n, d}$. However, because the internal gravity waves contain only a small fraction of the total available energy these can be calculated with a lower accuracy on a coarser grid with no loss of practical accuracy. The details of the vertical decoupling and evaluation of the number of corrected modes can be found in [4].

The idea to correct only fastest vertical modes has been proposed by Burridge [6] in the context of split-explicit scheme and afterwards it has been applied with success in different atmospheric models [4, 10, 12]. The space splitting approach has been proposed by Turkel and Zwas [25] in the context of shallow-water equations and developed in various articles $[4,16,21]$.

\subsection{Splitting the System (9) and Its Solution}

By eliminating the divergence in the last equation, the system (9) is reduced to Helmholtz equation

$$
G_{k}-\tau^{2} c_{k}^{2} \nabla^{2} G_{k}=-\tau c^{2} D_{k}^{*}+\tau^{2} c^{2} \nabla^{2} G_{k}^{*} \equiv F .
$$

Applying the approach tested by Tanguay and Robert in shallow water model [23], we modify slightly the third equation of the correction system by introducing the forth-order terms:

$$
\begin{gathered}
u_{k}+\tau G_{k x}=-\tau G_{k x}^{*}, v_{k}+\tau G_{k y}=-\tau G_{k y}^{*}, \\
G_{k}+\tau^{4} c_{k}^{4} G_{k_{x x y y}}+\tau c_{k}^{2} D_{k}=-\tau c_{k}^{2} \cdot D_{k}{ }^{*}-\tau^{4} c_{k}^{4} G_{k_{x x y y}}^{*} .
\end{gathered}
$$

The system (12) can be reduced to elliptic equation

$$
G_{k}-\tau^{2} c^{2} \nabla^{2} G_{k}+\tau^{4} c^{4} G_{k_{x x y y}}=F-\tau^{4} c^{4} G_{k_{x x y y}}^{*} \equiv \tilde{F} .
$$

The left-hand side of the last equation factorizes into two 1D operators

$$
\left(1-\tau^{2} c^{2} \partial_{x x}\right)\left(1-\tau^{2} c^{2} \partial_{y y}\right) G_{k}=\tilde{F}
$$

such that usual second order approximation of (14) leads to tridiagonal systems of linear algebraic equations, which can be effectively solved by direct Gelfand-Thomas algorithm [9].

Other theoretically economic methods for solution of (13) are iterative multigrid methods. These are also applicable to unmodified equation (11), but to obtain solution with required level of accuracy, starting from available initial guess (zero correction or previous step correction), the multigrid methods take approximately twice as much time as Gelfand-Thomas algorithm.

The final computations is quite simple: obtained corrections for the first five modes are transformed into physical corrections by the formulas inverse to (8) and one time step is completed by applying the diagnostic equations (3). We will refer to described 
scheme as SLSI-VH (semi-Lagrangian semi-implicit vertically-horizontally splitted) scheme.

Linear analysis showed that each of the vertical modes $k=1, \ldots, I$ corrected by formulas (12) is stable if

$$
\tau \leq 1 / f
$$

(note that the nonmodified system (19) has the same stability criterion). Each of uncorrected modes $k=I+1, \ldots, K$ required the stability condition

$$
\tau \leq h_{g} / \sqrt{2} c_{k} \sqrt{1+f^{2} h_{g}^{2} / 2 c_{k}^{2}}
$$

where $c_{k}$ is respective gravity-wave speed and $h_{g}$ is the horizontal grid step regarding gravity-waves discretization. Obviously, the strongest restriction on time step corresponds to the first vertical mode treated explicitly, which has the fastest gravity wave speed $c_{I+1}=c_{6}=\sqrt{R T_{0} \lambda_{6}} \approx 30 \mathrm{~m} / \mathrm{s}$ among other uncorrected modes. Comparing the criterions (6), (15) and (16) for $c_{6} \approx 30 \mathrm{~m} / \mathrm{s}$ and $h_{g}=112.5 \mathrm{~km}$, we conclude that the maximum allowable time step for SLSI-VH scheme is about 40 minutes.

Application of different time splitting techniques in the context of the atmospheric models was described in a number of papers (for instance, $[2,7,23,10]$ ). In the most cases these were schemes for shallow water equations. They showed very good performance in the context of Eulerian semi-implicit schemes, but the serious problems of losing accuracy for large time steps were detected in the context of semiLagrangian schemes, which permit to use the time steps above 30 minutes $[2,23,26]$.

We use the kind of time splitting proposed by Tanguay and Robert [23], who demonstrated that their scheme works well in shallow water model for time steps up to 40 minutes. They used horizontal grid with $190 \mathrm{~km}$ meshsize and respective time step, based on accuracy consideration, could be chosen greater than 60 minutes. However, the scheme starts breaking down at time steps on the order of 45 minutes primarily because of the nature of the truncation errors that are associated with splitting technique. Nevertheless, in the fine grid model with horizontal meshsize $75 \mathrm{~km}$ we may not want to choose time step in excess of $40 \mathrm{~min}$ because of both accuracy reasons and trajectory criterion (6). In the next section we show that in this case the SLSI-VH scheme has very good performance.

\section{Numerical Results}

In the first experiment, we evaluate the errors introduced because of vertical and horizontal splitting. Three versions of the semi-Lagrangian semi-implicit model (SLSI, SLSI-V and SLSI-VH) were run to produce 24-h forecasts on $97 \times 97$ point horizontal grid at each of 15 vertical levels. To solve the Helmholtz equation (11) in SLSI and SLSI-V schemes, the multigrid method by Dendy was used [8]. The rootmean-square height differences between SLSI and SLSI-V versions and SLSI-V and 
SLSI-VH were evaluated at the model vertical levels which correspond to pressure surfaces of 200, 500 and $1000 \mathrm{hPa}$ approximately. The results are shown in Table 1. The time steps used for these computations are shown in the first line of this Table. We see that these differences are very small for short time steps and they remain to be sufficiently small up to 40-min time step.

Table 1. Comparison of the three versions of SLSI scheme. The root-mean-square height differences are given in meters and time steps in minutes

\begin{tabular}{|c|c|c|c|c|c|}
\hline schemes, levels & $5 \mathrm{~min}$ & $10 \mathrm{~min}$ & $20 \mathrm{~min}$ & $30 \mathrm{~min}$ & $40 \mathrm{~min}$ \\
\hline SLSI/SLSI-V, 500hPa & 0.1 & 0.3 & 0.6 & 1.1 & 2.0 \\
\hline SLSI/SLSI-V, 1000hPa & 0.2 & 0.4 & 0.8 & 1.4 & 2.6 \\
\hline SLSI-V/SLSI-VH, 500hPa & 0.1 & 0.2 & 0.5 & 1.3 & 2.9 \\
\hline SLSI-V/SLSI-VH, 1000hPa & 0.1 & 0.3 & 0.7 & 1.7 & 3.8 \\
\hline
\end{tabular}

In order to verify the effectiveness and precision of the considered versions of SLSI scheme, the Eulerian leap-frog scheme was run on the same spatial grid and with time step $\tau=1 \mathrm{~min}$ to produce "exact" numerical solution. The root-meansquare differences between 24-h geopotential forecasting fields produced by SLSI, SLSI-V, SLSI-VH schemes and leap-frog scheme are presented in Table 2. We can see that these differences are greater than ones in Table 1 because of different spatial and time truncation errors in semi-Lagrangian semi-implicit and Eulerian explicit models. However these differences are still at an acceptable level.

Table 2. Comparative characteristics of the different schemes. $\tau$ - time step in minutes used in indicated model; $\delta_{200}, \delta_{500}, \delta_{1000}$ - root-mean-square height differences in meters between the 24-h forecasts produced by chosen scheme and leap-frog scheme at the heights $200 \mathrm{hPa}$, $500 \mathrm{hPa}$ and $1000 \mathrm{hPa}$, respectively; $T_{C P U}$ - computational time cost of one forecast regarding leap-frog forecast time

\begin{tabular}{|c|c|c|c|c|c|}
\hline scheme & $\tau$ & $\delta_{200}$ & $\delta_{500}$ & $\delta_{1000}$ & $T_{C P U}$ \\
\hline leap-frog & 1 & 0 & 0 & 0 & 1 \\
\hline SLSI & 40 & 5.3 & 5.2 & 4.8 & 0.16 \\
\hline SLSI-V & 40 & 5.4 & 5.2 & 4.9 & 0.14 \\
\hline SLSI-VH & 40 & 5.6 & 5.3 & 5.2 & 0.11 \\
\hline
\end{tabular}

Finally, the thirty 24-h forecasts were calculated by SLSI and SLSI-VH schemes with 40-min time step on the same spatial grid, whose center is near Porto Alegre city $\left(30^{0} \mathrm{~S}, 52^{0} \mathrm{~W}\right)$. The initial and boundary conditions were obtained from objective analysis and medium range global forecasts of NCEP. Two mean objective scores of the geopotential forecasts were calculated at different vertical levels: the root-meansquare differences in meters between 24-h forecasts and NCEP analysis and the correlation coefficient between observed and forecast changes. Also the leap-frog scheme with 1-min time step was run to compare the relative accuracy of the different schemes. The results of these estimations are shown in Table 3. Taking into account that this version of the baroclinic model is quasi adiabatic, obtained evaluations can be considered successful and it confirms the validity of applied splitting techniques. 
Table 3. Mean objective scores of the 24 -h geopotential forecasts. $\varepsilon_{200}, \varepsilon_{500}, \varepsilon_{1000}$ - rootmean-square height differences in meters between the analysis and the 24-h forecasting fields at the heights $200 \mathrm{hPa}, 500 \mathrm{hPa}$ and $1000 \mathrm{hPa}$, respectively; $r_{200}, r_{500}, r_{1000}-$ correlation coefficients (nondimensional) between the analysis and 24-h forecasting fields at the heights $200 \mathrm{hPa}, 500 \mathrm{hPa}$ and $1000 \mathrm{hPa}$, respectively

\begin{tabular}{|c|c|c|c|c|c|c|}
\hline scheme & $\varepsilon_{200}$ & $r_{200}$ & $\varepsilon_{500}$ & $r_{500}$ & $\varepsilon_{1000}$ & $r_{1000}$ \\
\hline leap-frog & 29 & 0.89 & 20 & 0.91 & 25 & 0.87 \\
\hline SLSI & 29 & 0.90 & 21 & 0.92 & 25 & 0.86 \\
\hline SLSI-VH & 28 & 0.90 & 21 & 0.92 & 26 & 0.86 \\
\hline
\end{tabular}

\section{Conclusion}

An efficient semi-Lagrangian semi-implicit time and space splitted algorithm was developed for adiabatic hydrostatic atmospheric model. It permits to use the time steps up to 40 minutes and required amount of computations is directly proportional to the number of spatial grid points. Computational efficiency of developed algorithm and its accuracy in 24-h forecasting of meteorological fields were confirmed by numerical tests carried out with actual meteorological data.

\section{References}

1. Arakawa A., Konor C.S.: Vertical differencing of the primitive equations based on the Charney-Phillips grid in hybrid $\sigma-p$ vertical coordinates. Mon. Wea. Rev. 124 (1996) 511-528.

2. Bates J.R.: An efficient semi-Lagrangian and alternating direction implicit method for integrating the shallow water equations. Mon. Wea. Rev. 112 (1984) 2033-2047.

3. Benoit R., Desgagne M., Pellerin P., Pellerin S., Chartier Y., Desjardins S.: The canadian MC2: a semi-Lagrangian, semi-implicit wideband atmospheric model suited for finescale process studies and simulation. Mon. Wea. Rev. 125 (1997) 2382-2415.

4. Bourchtein A.: Semi-Lagrangian semi-implicit space splitting regional baroclinic atmospheric model. Appl. Numer. Math. 40 (2002) 307-326.

5. Bourchtein A., Kadychnikov V.: Well-posedness of the initial value problem for vertically discretized hydrostatic equations. SIAM J. Numer. Anal. (2003, to appear).

6. Burridge D.M.: A split semi-implicit reformulation of the Bushby-Timpson 10 level model. Quart. J. Roy. Meteor. Soc. 101 (1975) 777-792.

7. Cohn S.E., Dee D., Isaacson E., Marchesin D., Zwas G.: A fully implicit scheme for the barotropic primitive equations. Mon. Wea. Rev. 113 (1985) 436-448.

8. Dendy J.E.: Black box multigrid. J. Comput. Phys. 48 (1982) 366-386.

9. Godunov S. K., Ryabenkii V. S.: Difference Schemes: An Introduction to the Underlying Theory. Elsevier, New York (1987).

10. Kadychnikov V., Losev V.: Application of the alternating direction implicit method to the numerical regional weather forecast. Meteor. and Hydrol. 9 (1991) 26-33.

11. Kiehl J.T., Hack J.J., Bonan G.B., Boville B.A., Williamson D.L., Rasch P.J.: The National Center for Atmospheric Research Community Climate Model: CCM3. J. Climate 11 (1998) 1131-1149. 
12. Leslie L.M., Purser R.J.: Three-dimensional mass-conservating semi-Lagrangian scheme employing forward trajectories. Mon. Wea. Rev. 123 (1995) 2551-2566.

13. Mcdonald A.: Accuracy of multiply-upstream semi-Lagrangian advective schemes II. Mon. Wea. Rev. 115 (1987) 1446-1450.

14. Mesinger F., Arakawa A.: Numerical Methods Used in Atmospheric Models. GARP Publications Series, WMO/ICSU Joint Organizing Committee, Geneva (1976).

15. Moorthi S.: NWP Experiments with a gridpoint semi-Lagrangian semi-implicit global model at NCEP. Mon. Wea. Rev. 125 (1997) 74-98.

16. Navon I.M., de Villiers R.: The application of the Turkel-Zwas explicit large time-step scheme to a hemispheric barotropic model with constraint restoration. Mon. Wea. Rev. 115 (1987) 1036-1051.

17. Pudykiewicz J., Benoit R., Staniforth A.: Preliminary results from a partial LRTAP model based on an existing meteorological forecast model. Atmos.-Ocean 23 (1985) 267-303.

18. Ritchie H., Temperton C., Simmons A., Hortal M., Davies T., Dent D., Hamrud M.: Implementation of the semi-Lagrangian method in a high-resolution version of the ECMWF forecast model. Mon. Wea. Rev. 123 (1995) 489-514.

19. Robert A.: A stable numerical integration scheme for the primitive meteorological equations. Atmos.-Ocean 19 (1981) 35-46.

20. Robert A., Yee T.L., Ritchie H.: A semi-Lagrangian and semi-implicit numerical integration scheme for multilevel atmospheric models. Mon. Wea. Rev. 113 (1985) 388394.

21. Song Y., Tang T.: Staggered Turkel-Zwas schemes for two-dimensional shallow-water equations. Mon. Wea. Rev. 122 (1994) 223-234.

22. Staniforth A., Côté J.: Semi-Lagrangian integration schemes for atmospheric models - A review. Mon. Wea. Rev. 119 (1991) 2206-2223.

23. Tanguay M., Robert A.: Elimination of the Helmholtz equation associated with the semiimplicit scheme in a grid point model of the shallow water equations. Mon. Wea. Rev. 114 (1986) 2154-2162.

24. Tanguay M., Robert A., Laprise R.: A semi-implicit semi-Lagrangian fully compressible regional forecast model. Mon. Wea. Rev. 118 (1990) 1970-1980.

25. Turkel E., Zwas G.: Explicit large-time-step schemes for the shallow-water equations. In: Vichnevetsky R, Stepleman R.S. (eds.): Advances in Computer Methods for Partial Differential Equations, Lehigh University (1979) 65-69.

26. Yakimiw E., Robert A.: Accuracy and stability analysis of a fully implicit scheme for the shallow water equations. Mon. Wea. Rev. 114 (1986) 240-244. 